\title{
Influence of composition on the unipolar electric fatigue of $\mathrm{Ba}\left(\mathrm{Zr}_{0.2} \mathrm{Ti}_{0.8}\right) \mathrm{O}_{3}-\left(\mathrm{Ba}_{0.7} \mathrm{Ca}_{0.3}\right) \mathrm{TiO}_{3}$ lead-free piezoceramics
}

\author{
Virginia Rojas $^{1}$ (D) | Jurij Koruza ${ }^{1}$ (D) | Eric A. Patterson ${ }^{2}$ (D) | Matias Acosta $^{1}$ (D) | \\ Xijie Jiang1 $^{1}$ (i) | Na Liu ${ }^{1}$ | Christian Dietz ${ }^{1}$ | Jürgen Rödel ${ }^{1}$
}

\begin{abstract}
${ }^{1}$ Institute of Materials Science, Technische Universität Darmstadt, Darmstadt, Germany

${ }^{2}$ Materials Science and Technology Division, U.S. Naval Research Laboratory, Washington, District of Columbia
\end{abstract}

\section{Correspondence}

Jurij Koruza, Institute of Materials

Science, Technische Universität

Darmstadt, Darmstadt, Germany.

Email: koruza@ceramics.tu-darmstadt.de

\begin{abstract}
The lead-free $(1-x) \mathrm{Ba}\left(\mathrm{Zr}_{0.2} \mathrm{Ti}_{0.8}\right) \mathrm{O}_{3}-x\left(\mathrm{Ba}_{0.7} \mathrm{Ca}_{0.3}\right) \mathrm{TiO}_{3}$ system is considered as promising candidate for the replacement of lead-based piezoceramics in actuation applications, during which electric fatigue is a major concern. This issue was addressed in this work, where the unipolar fatigue resistance of three $(1-x) \mathrm{Ba}$ $\left(\mathrm{Zr}_{0.2} \mathrm{Ti}_{0.8}\right) \mathrm{O}_{3}-x\left(\mathrm{Ba}_{0.7} \mathrm{Ca}_{0.3}\right) \mathrm{TiO}_{3}$ compositions with different crystallographic structures (rhombohedral, orthorhombic, and tetragonal) was evaluated. Strain asymmetry and development of an internal bias field were observed in all compositions. The decrease in the remanent polarization and the large signal piezoelectric coefficient after $10^{7}$ unipolar cycles was found to lie between $6 \%$ $12 \%$ and $2 \%-13 \%$, respectively. The most pronounced fatigue was observed for the orthorhombic composition, which has the largest extrinsic contribution to strain. On the other hand, the best fatigue resistance was observed for the tetragonal composition, which has a predominantly intrinsic strain response. The correlation of fatigue resistance with strain mechanism was corroborated with determination of the Rayleigh parameters and changes in the domain morphology after cycling as confirmed by piezoresponse force microscopy.
\end{abstract}

K E Y W O R D S

cyclic fatigue, domains, fatigue, lead-free ceramics, piezoelectric materials/properties

\section{1 | INTRODUCTION}

The ferroelectrics that have been widely used for piezoelectric actuator devices over the past 50 years are lead-containing materials based on lead zirconate titanate $\left(\mathrm{PbZr}_{x} \mathrm{Ti}_{1-x} \mathrm{O}_{3}\right.$, PZT). However, there has been a growing concern related to the hazardous effect of toxic lead on the environment and human health. This concern was reinforced by governmental regulations that increasingly restrict the use of lead in ceramic components. ${ }^{1}$ As a result, the focus of the piezoelectric research community was shifted toward the development of new lead-free materials. ${ }^{2,3}$ Prominent candidates, considered to replace lead-containing compositions in applications, are based on potassium sodium niobate $(\mathrm{KNN}),{ }^{4-6}$ sodium bismuth titanate (NBT), ${ }^{7,8}$ and barium titanate (BT) ${ }^{9,10}$ Nonetheless, the investigations of their fatigue resistance, that is, degradation of properties during electromechanical cycling, are relatively scarce and could hinder their implementation. ${ }^{11-13}$

Fatigue studies are of great importance for the industrial application of piezoelectric actuators since it is one of the major degradation mechanisms of piezoelectric properties. $^{14,15}$ Many authors have studied the effects of electric field cycling on lead-based ferroelectrics. ${ }^{16-19}$ In general, asymmetries and offsets in the polarization and strain hysteresis curves, decrease in polarization, and even mechanical degradation were reported. Moreover, mechanical failure due to cracks was observed as a result of electric 


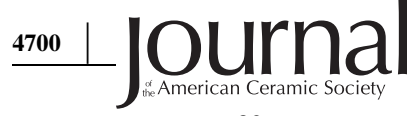

fatigue in PZT. ${ }^{20}$ Defects already present in the material, such as microcracks, pores, and electrode edges, are thought to constitute stress concentration points and serve as the origin for crack growth when submitted to electric cycling. ${ }^{21,22}$ On the other hand, point defects such as oxygen vacancies and other charge carriers are also susceptible to the applied electric field and migrate during cycling. The migration of such defects toward the grain boundaries or electrodes can hinder domain wall movement, which affects the overall magnitude of electromechanical properties of the material. ${ }^{20,23-25}$ The deterioration of properties caused by electric fatigue might change with the different loading scenarios, ${ }^{17}$ which include unipolar, ${ }^{26,27}$ bipolar, ${ }^{24}$ or sesquipolar ${ }^{28}$ cycling, and with or without compressive preloads. ${ }^{29}$ Bipolar electric cycling has a more severe effect than unipolar cycling in the degradation of properties and its effects are relevant for a complete characterization of the material. However, as actuators are generally driven with unipolar electric fields, the study of the unipolar cycling scenario is essential from an application point of view.

The high piezoelectric properties of the lead-free $(1-x)$ $\mathrm{Ba}\left(\mathrm{Zr}_{0.2} \mathrm{Ti}_{0.8}\right) \mathrm{O}_{3}-x\left(\mathrm{Ba}_{0.7} \mathrm{Ca}_{0.3}\right) \mathrm{TiO}_{3} \quad(\mathrm{BZT}-x \mathrm{BCT})$ system, most notably at room temperature, $d_{33}$ of $620 \mathrm{pC} / \mathrm{N}$ and a $d_{33}^{*}$ of $1140 \mathrm{pm} / \mathrm{V}$ were first reported by Liu and Ren in 2009. ${ }^{9}$ In addition to their high piezoelectric coefficient, these materials exhibit a high blocking stress $\left(\sigma_{\mathrm{b}}\right)$ of about $-64 \mathrm{MPa}$ at $2 \mathrm{kV} / \mathrm{mm},{ }^{30}$ and low coercive fields $\left(\mathrm{E}_{\mathrm{C}}\right.$ below $0.5 \mathrm{kV} / \mathrm{mm}) .{ }^{31}$ It should be noted that these values exceed the performance of commercially available soft PZT. ${ }^{32}$ The bipolar fatigue degradation of BZT-50BCT $\left(\mathrm{Ba}_{0.85} \mathrm{Ca}_{0.15} \mathrm{Ti}_{0.9} \mathrm{Zr}_{0.1} \mathrm{O}_{3}\right)$ and a second composition with less $\mathrm{Ca}$ and $\mathrm{Zr}\left(\mathrm{Ba}_{0.92} \mathrm{Ca}_{0.08} \mathrm{Ti}_{0.945} \mathrm{Zr}_{0.055} \mathrm{O}_{3}\right)$ were recently studied by Zhang et al. ${ }^{33}$ Both compositions were shown to have higher resistance to bipolar fatigue at room temperature, as compared to soft PZT and NBT-BT materials. The composition with a lower amount of $\mathrm{Ca}$ and $\mathrm{Zr}$ exhibited a weaker domain wall pinning effect, but exhibited signs of larger mechanical degradation by microcracking due to the presence of large and irregular pores. Therefore, its overall polarization loss was higher than that of BZT50BCT. In a subsequent work, Zhang et al. ${ }^{34}$ investigated the effects of unipolar cycling in BZT-50BCT and demonstrated that the unipolar fatigue effects on the material were comparable to those of soft PZT. The degradation of the properties was proposed to originate primarily from an accumulation of charge carriers at grain boundaries. ${ }^{34}$

The majority of studies on BZT- $x$ BCT materials focus on the composition where $\mathrm{x}=0.50$, although other compositions also possess excellent properties. For instance, there is a higher blocking stress for BZT-42BCT, ${ }^{30}$ improved temperature stability of $d_{33}^{*}$ in BZT-60BCT, ${ }^{35}$ and higher $\mathrm{T}_{\mathrm{c}}$ for compositions with less $\mathrm{Zr}$ and $\mathrm{Ca}$, such as in $(\mathrm{Ba}$ $\left.\left(\mathrm{Zr}_{0.15} \mathrm{Ti}_{0.85}\right) \mathrm{O}_{3}-x\left(\mathrm{Ba}_{0.8} \mathrm{Ca}_{0.2}\right) \mathrm{TiO}_{3}\right){ }^{36}$ Moreover, the strain responses of different compositions were shown to be dominated by different mechanisms, which could have significant impacts on their cyclic degradation characteristics. ${ }^{37,38}$ The aim of this study is therefore to investigate the unipolar fatigue behavior of BZT- $x$ BCT ceramics with different compositions with distinct crystal structures. Strain and polarization curves were evaluated up to $10^{7}$ electric field cycles. The results are corroborated with Rayleigh analysis and domain configuration imaging using piezoresponse force microscopy before and after cycling.

\section{EXPERIMENTAL PROCEDURE}

Three compositions of the $(1-x) \mathrm{Ba}\left(\mathrm{Zr}_{0.2} \mathrm{Ti}_{0.8}\right) \mathrm{O}_{3}$ - $\mathrm{x}$ $\left(\mathrm{Ba}_{0.7} \mathrm{Ca}_{0.3}\right) \mathrm{TiO}_{3}$ (BZT- $\left.x \mathrm{BCT}\right)$ system with $\mathrm{x}=0.40,0.50$, and 0.60 , hereafter referred to as $40 \mathrm{BCT}(\mathrm{R}), 50 \mathrm{BCT}(\mathrm{O})$, and $60 \mathrm{BCT}(\mathrm{T})$, were prepared by the solid-state synthesis. Note that the letter in parentheses denotes the crystal structure at room temperature: rhombohedral (R), orthorhombic $(\mathrm{O})$, and tetragonal $(\mathrm{T})$, as determined by X-ray diffraction (D8 Advance, Bruker Inc., Karlsruhe, Germany). Powders of $\mathrm{BaCO}_{3}$ (99.8\%), $\mathrm{CaCO}_{3}$ (99.5\%), $\mathrm{ZrO}_{2}$ (99.5\%), and $\mathrm{TiO}_{2}(99.6 \%)$ were mixed according to the corresponding stoichiometric formula and milled for 5 hours in a planetary mill using ethanol and $\mathrm{ZrO}_{2}$ milling media. The suspension was then dried and the powders were calcined at $1300^{\circ} \mathrm{C}$ for 2 hours. After calcination the powders were again milled, dried, and formed into disks by uniaxial hand pressing in a 10-mm die, followed by cold isostatic pressing (CIP 100 E, Paul-Otto Weber GmbH, Remshalden, Germany) at $300 \mathrm{MPa}$. Sintering was performed at $1500^{\circ} \mathrm{C}$ for 2 hours, with a heating rate of $5^{\circ} \mathrm{C} / \mathrm{min}$. The sintered pellets were ground to a thickness of about $0.7 \mathrm{~mm}$ and silver electrodes (silver paste, Gwent Group Ltd., Gwent, United Kingdom) were painted and burnt-in at $400^{\circ} \mathrm{C}$. Subsequent annealing treatments after completing the unipolar cycling measurements were done at $400^{\circ} \mathrm{C}$ for 2 hours. Density measurements were carried out using an Archimedes balance and grain size was calculated with the linear intercept method from scanning electron microscopy (SEM) micrographs.

For the characterization of piezoelectric properties, a modified Sawyer-Tower circuit with an optical displacement sensor (Model D63, Philtec Inc., Annapolis, USA) was used. The electric field cycling tests were carried out using unipolar triangular waveforms with an amplitude of five times the coercive field $\left(\mathrm{E}_{\mathrm{C}}\right)$ and a frequency of $50 \mathrm{~Hz}$. A maximum of $10^{7}$ cycles were performed and a measurement of bipolar and unipolar polarization and strain was done after every cycling decade at $5 \cdot \mathrm{E}_{\mathrm{C}}$ but at a frequency of $1 \mathrm{~Hz}$. The first $10^{5}$ cycles were done on the modified Sawyer-Tower circuit, which was also used for 
characterization. The electric cycling from $10^{5}$ to $10^{7}$ cycles, equivalent to $\sim 55$ hours of cycling, was carried out using a Sawyer-Tower setup designed for longer running high-voltage experiments.

All initial electrical characterizations were performed on freshly annealed samples, without previous DC poling. A total of three samples of each composition were submitted to unipolar cycling. The mean value of the internal bias field, strain asymmetry, and $d_{33}^{*}$ values are reported throughout this work, while the error bars represent the standard deviation. Rayleigh analysis was done by measuring the dielectric permittivity as a function of AC electric field, using an impedance analyzer with a high voltage interface (Alpha-A analyzer and High Voltage Booster HVB300, Novocontrol technologies, Montabaur, Germany) at $100 \mathrm{~Hz}$ with electric field amplitudes between 0 and $0.06 \mathrm{kV} / \mathrm{mm}$.

The domain structure of polished surfaces of samples before and after cycling was observed using vector piezoresponse force microscopy (PFM). ${ }^{39}$ The polished samples were first imaged in the annealed state. The position of each image was recorded on an optical microscope picture of the polished surfaces. Subsequently, silver electrodes (Silberleitlack, Ferro GmbH, Frankfurt, Germany) were painted and dried at room temperature followed by the application of the $10^{7}$ unipolar cycles. Afterward the electrodes were removed using acetone. The samples were then carefully repositioned on the PFM holder according to the images taken in the optical microscope in order to view the same area as before cycling. All PFM images were taken within 24 hours after the cycling was finished.

A Cypher atomic force microscope (Asylum Research, Santa Barbara, CA, USA) was used to perform the PFM measurements. The nominal force constant and first free resonance frequency of the conductive cantilevers ASYELEC-01 (Asylum Research, Santa Barbara, CA, USA) were $k=2 \mathrm{~N} / \mathrm{m}$ and $f=70 \mathrm{kHz}$, respectively. The contact resonance frequencies for the cantilever vibration on the BZT$x \mathrm{BCT}$ samples were $f_{\mathrm{CR}, \mathrm{v}} \approx 300 \mathrm{kHz}$ and $f_{\mathrm{CR}, \mathrm{I}} \approx 870 \mathrm{kHz}$ for the vertical and lateral vibration, respectively. The excitation frequencies for both directions were set slightly below the respective contact resonance frequencies in such way that the vibrational amplitudes were approximately $30 \%$ of the peak amplitude. A drive amplitude in both directions in the range of $2-4 \mathrm{~V}$, a scan rate of $0.3 \mathrm{~Hz}$ (tip velocity $12 \mu \mathrm{m} / \mathrm{s}$ ) and a digital resolution of $256 \times 256$ pixel were applied. Topography images were first-order flattened, whereas amplitude and phase images remained untreated.

\section{3 | RESULTS}

The three investigated compositions, 40BCT(R), 50BCT $(\mathrm{O})$, and $60 \mathrm{BCT}(\mathrm{T})$, have different crystallographic

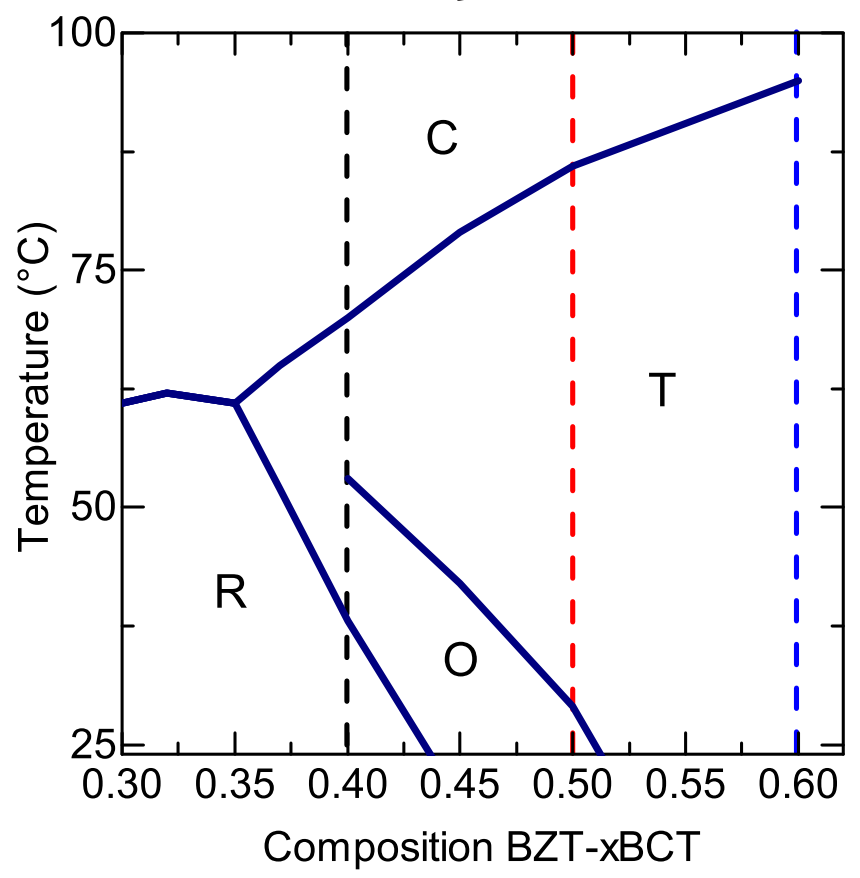

FIGURE 1 Phase diagram of the BZT- $x$ BCT system, modified after Ref. 35, where the dashed lines indicate the compositions selected for this study [Color figure can be viewed at wileyonlinelibrary.com]

structures at room temperature, in agreement with previous works. ${ }^{35,40}$ Figure 1 presents a modified version of the phase diagram proposed by Acosta et al. ${ }^{35}$ indicating that the crystallographic structure of the $40 \mathrm{BCT}(\mathrm{R})$ is rhombohedral at room temperature and transforms to orthorhombic at $38^{\circ} \mathrm{C}$, to tetragonal at $53^{\circ} \mathrm{C}$, and to cubic at $70^{\circ} \mathrm{C}$. The $50 \mathrm{BCT}(\mathrm{O})$ is in the orthorhombic phase at room temperature, transitions into tetragonal phase at $29^{\circ} \mathrm{C}$, and to the cubic phase at $86^{\circ} \mathrm{C}$. On the other hand, the tetragonal composition $60 \mathrm{BCT}(\mathrm{T})$ has no phase transitions between room temperature and the Curie temperature at $95^{\circ} \mathrm{C}$, where it transforms into the cubic structure. Since the compositions have different crystal structures, they also show differences in their ferroelectric properties. ${ }^{37}$ The differences originate from different possible directions for spontaneous polarization ( 6 for tetragonal, 8 for rhombohedral, and 12 for orthorhombic), different ferroelastic domains $\left(90^{\circ}\right.$ domains for tetragonal, $109^{\circ}$ and $71^{\circ}$ for rhombohedral, and $60^{\circ}$ and $120^{\circ}$ for orthorhombic), different lattice distortions, and different proximities of room temperature to their closest phase transitions. The corresponding characteristic values of $E_{C}$ and $P_{r}$, as well as sample densities and grain sizes, are presented in Table 1 . The densities of all samples were above $95 \%$ and grain sizes were similar across the different compositions.

Figure 2 displays the bipolar strain and polarization hysteresis curves in the initial annealed state, after $10^{7}$ unipolar cycles, and after subsequent thermal annealing after cycling. 


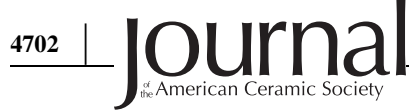

TABLE 1 Characteristic microstructural parameters and ferroelectric values of the investigated BZT- $x$ BCT compositions

\begin{tabular}{lccc} 
& 40BCT(R) & 50BCT(O) & 60BCT(T) \\
\hline Absolute density $\left(\mathrm{g} / \mathrm{cm}^{3}\right)$ & $5.68 \pm 0.10$ & $5.48 \pm 0.07$ & $5.47 \pm 0.01$ \\
\hline Relative density $(\%)$ & $97.3 \pm 1.8$ & $95.1 \pm 1.3$ & $95.9 \pm 0.1$ \\
\hline Mean grain size $(\mu \mathrm{m})$ & $28.1 \pm 5.9$ & $28.9 \pm 4.6$ & $25.0 \pm 4.5$ \\
$\mathrm{E}_{\mathrm{C}}(\mathrm{kV} / \mathrm{mm})$ & 0.20 & 0.25 & 0.50 \\
$\mathrm{P}_{\mathrm{r}}\left(\mu \mathrm{C} / \mathrm{cm}^{2}\right)$ & $10.32 \pm 0.33$ & $12.31 \pm 0.86$ & $11.43 \pm 0.11$ \\
\hline
\end{tabular}

The curves were measured at a cycling field of $5 \cdot \mathrm{E}_{\mathrm{C}}$ and are presented with the $x$-axis normalized to multiples of the coercive field value in order to evaluate changes to the hysteresis in a more direct manner. All compositions exhibit fatigue behavior, demonstrated by the asymmetric strain curves and the shift of the polarization curves along the abscissa axis toward the negative side of the applied electric field. Both effects suggest that unipolar cycling induced an internal electric bias field. The strain asymmetry is more pronounced in the orthorhombic 50BCT $(\mathrm{O})$ composition and is less prominent in the rhombohedral and tetragonal compositions. The effect of fatigue is also reflected in the unipolar strain curves, evident by the lowering of the maximum achieved strain for all compositions. Thermal annealing of the fatigued samples at $400^{\circ} \mathrm{C}$ for 2 hours, however, resulted in the full recovery of the electromechanical properties, which indicates that no permanent degradation was induced during the unipolar electric field cycling.
In order to quantitatively evaluate the effect of the unipolar cycling, the internal bias field $\left(\mathrm{E}_{\mathrm{ib}}\right)$ and the strain asymmetry $\left(\gamma_{S}\right)$ were calculated for each decade of the cycling using the following equations ${ }^{26}$ :

$$
\begin{aligned}
& E_{\mathrm{ib}}=-\frac{E_{\mathrm{C}}^{+}+E_{\mathrm{C}}^{-}}{2} \\
& \gamma_{\mathrm{S}}=\frac{\Delta S^{+}-\Delta S^{-}}{\Delta S^{+}+\Delta S^{-}}
\end{aligned}
$$

Here $\mathrm{E}_{\mathrm{C}}{ }^{+}$and $\mathrm{E}_{\mathrm{C}}{ }^{-}$are the positive and negative coercive fields, and $\Delta \mathrm{S}^{+}$and $\Delta \mathrm{S}^{-}$correspond to the total strain, from most negative to most positive value, at each side of the bipolar strain curve. These results are shown in Figure 3, along with the trend in remanent polarization normalized to the first cycle. A gradual increase of the internal bias field is observed with increasing cycling. With the exception of the first cycle of the 60BCT(T), the bias field is positive in all three compositions, following the polarity of the applied unipolar cycles and shifting the center of the hysteresis loops to the negative applied field direction. While the $\mathrm{E}_{\mathrm{ib}}$ in rhombohedral 40BCT(R) stabilizes after $10^{3}$ cycles, the $\mathrm{E}_{\mathrm{ib}}$ in the orthorhombic 50BCT(O) continues to increase gradually throughout the investigated cycles. The bias field for the tetragonal $60 \mathrm{BCT}(\mathrm{T})$ remains close to zero up to $10^{5}$ cycles, after which it increases to the same level as the one of the $50 \mathrm{BCT}(\mathrm{O})$. However, since the compositions have different coercive fields, the comparison should be
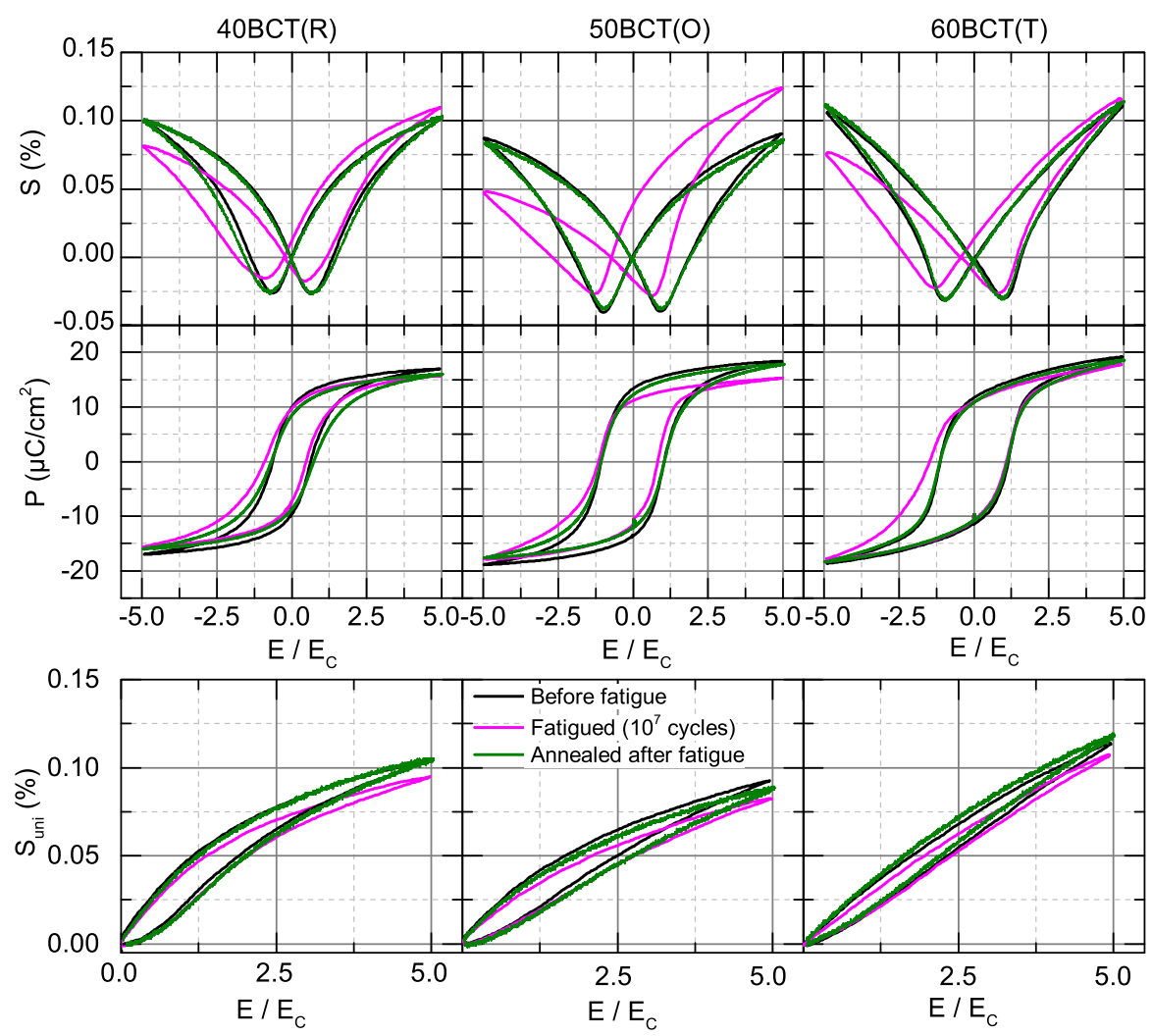

F IGURE 2 Bipolar strain and polarization and unipolar strain hysteresis curves as a function of the normalized electric field for the three investigated BZT$x \mathrm{BCT}$ compositions, 40BCT(R), 50BCT $(\mathrm{O})$, and $60 \mathrm{BCT}(\mathrm{T})$, before cycling (black), after $10^{7}$ unipolar cycles (pink), and after subsequent thermal annealing (green) [Color figure can be viewed at wileyonlinelibrary.com] 


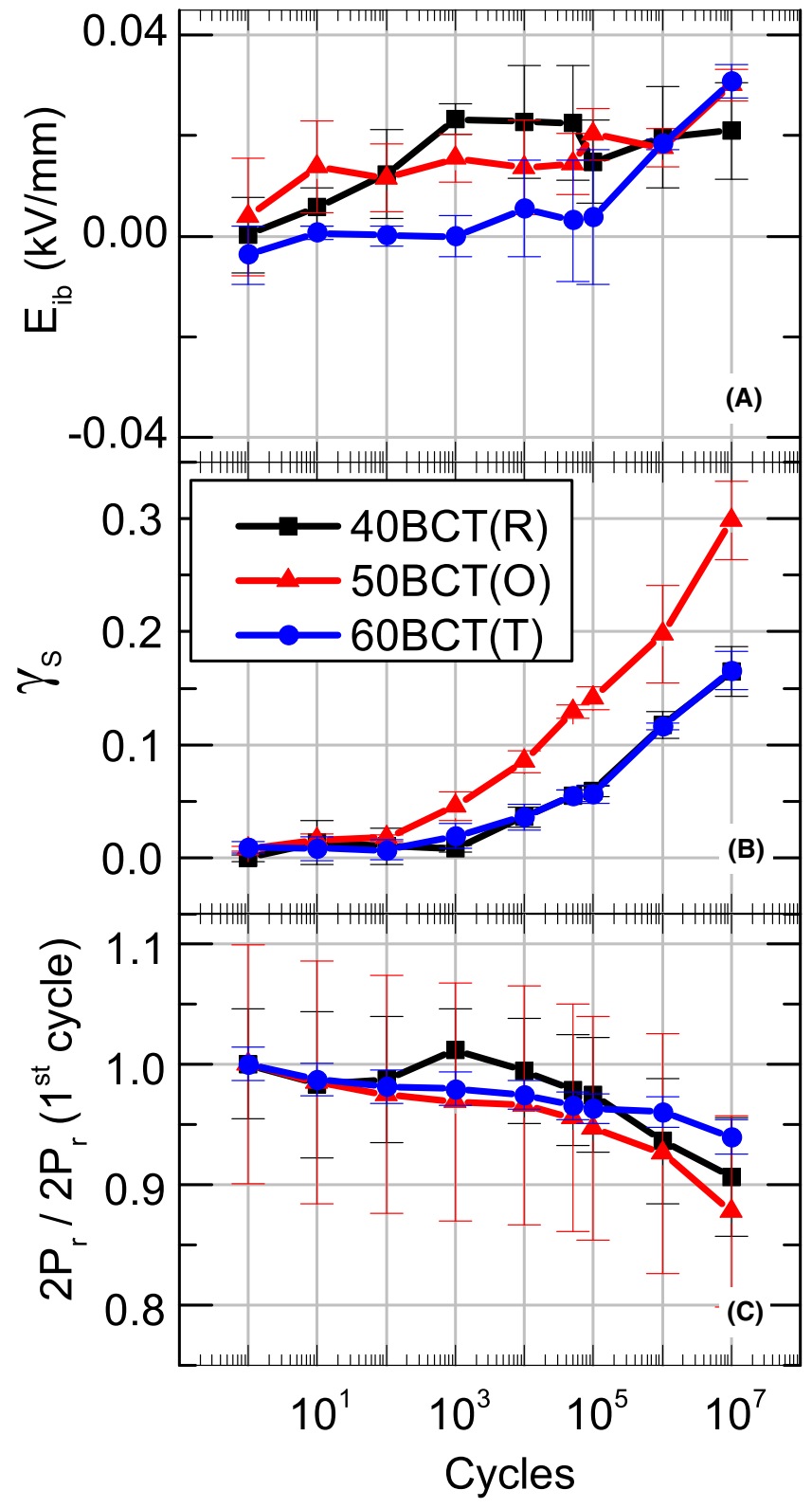

FIGURE 3 (A) Internal bias field ( $\left.\mathrm{E}_{\mathrm{ib}}\right)$, (B) strain asymmetry $\left(\gamma_{S}\right)$, and $(C)$ remanent polarization $\left(2 \mathrm{P}_{\mathrm{r}}\right)$ normalized by the first cycle as a function of unipolar cycles. The solid lines are only guides to the eye [Color figure can be viewed at wileyonlinelibrary.com]

done using the relative internal bias field $\left(\mathrm{E}_{\mathrm{ib}-\mathrm{rel}}\right)$, which is defined here as:

$$
E_{\mathrm{ib}-\mathrm{rel}}=\frac{E_{\mathrm{ib}}}{E_{\mathrm{C}}} \times 100 \%
$$

The $\mathrm{E}_{\mathrm{ib}-\text { rel }}$ is comparatively larger for the compositions with a low $\mathrm{E}_{\mathrm{C}}$ (i.e., $40 \mathrm{BCT}(\mathrm{R})$ and $50 \mathrm{BCT}(\mathrm{O})$ ). At $10^{7}$ unipolar cycles the $\mathrm{E}_{\mathrm{ib}-\mathrm{rel}}$ is $11 \%$ for the $40 \mathrm{BCT}(\mathrm{R})$ and $12 \%$ for $50 \mathrm{BCT}(\mathrm{O})$, while a lower $\mathrm{E}_{\mathrm{ib} \text {-rel }}$ of $6 \%$ is formed in the $60 \mathrm{BCT}(\mathrm{T})$ composition with its two times higher $\mathrm{E}_{\mathrm{C}}$.

The strain asymmetry as a function of the number of cycles is presented in Figure 3B, where an exponential increase is observed. The effect on the strain asymmetry seems to be much more pronounced in orthorhombic 50BCT(O), which after the initial $10^{2}$ cycles increases at a higher rate than the other two compositions.

Figure $3 \mathrm{C}$ shows the remanent polarization normalized by the first cycle remanent polarization value for all samples. For the first $10^{4}$ cycles, all compositions show a decrease between $2 \%$ and $4 \%$ of their initial value, while after $10^{5}$ cycles the decrease becomes more pronounced. The error bars in this plot are exaggerated by the normalization process. After $10^{7}$ cycles, the remanent polarization is reduced by about $6 \%$ for the $60 \mathrm{BCT}(\mathrm{T}), 9 \%$ for the $40 \mathrm{BCT}(\mathrm{R})$, and $12 \%$ for the $50 \mathrm{BCT}(\mathrm{O})$.

The effect of unipolar cycling on strain output is evaluated using the large signal piezoelectric coefficient $d_{33}^{*}$, also referred to as normalized strain. This coefficient is defined as $d_{33}^{*}=S_{\text {uni }}^{\max } / E^{\max }$, where $S_{\text {uni }}{ }^{\max }$ is the unipolar strain at the corresponding electric field $E^{\max }$. Figure $4 A$ depicts the absolute $d_{33}^{*}$ values of all three compositions as a function of the number of unipolar cycles, while Figure 4B features the $d_{33}^{*}$ normalized by the first cycle value. All compositions present an overall reduction of $d_{33}^{*}$ with increasing unipolar cycles. The most stable response is observed for

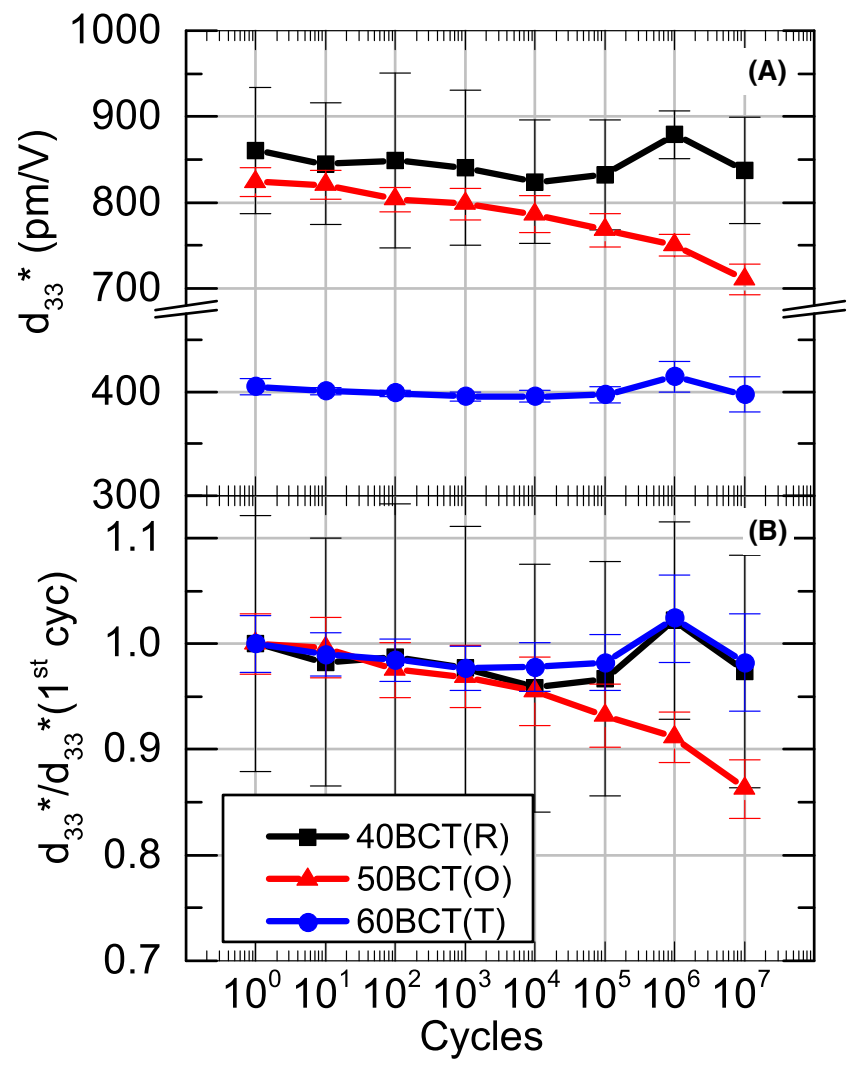

FIGURE 4 (A) Large signal $d_{33}^{*}$ calculated from unipolar curves at $0.8 \mathrm{kV} / \mathrm{mm}$, and (B) normalized $d_{33}^{*}$ as a function of the number of cycles. The solid lines are only guides to the eye [Color figure can be viewed at wileyonlinelibrary.com] 
the tetragonal $60 \mathrm{BCT}(\mathrm{T})$ with values of approximately $400 \mathrm{pm} / \mathrm{V}$ during the entire cycling period and a total reduction of the value of only about $2 \%$ after $10^{7}$ cycles. The rhombohedral $40 \mathrm{BCT}(\mathrm{R})$ has a similarly small decrease of $d_{33}^{*}$ of about $3 \%$ at $10^{7}$ cycles, but the scattering of the values taken during the cycling is larger. The slight $d_{33}^{*}$ increase observed at $10^{6}$ cycles for both $40 \mathrm{BCT}$ $(\mathrm{R})$ and $60 \mathrm{BCT}(\mathrm{T})$ in Figure 4A,B could be correlated with change of the cycling high voltage setup (described in the Experimental Procedure section). However, as these deviations are within the measurement errors they will not be considered further. On the other hand, the orthorhombic $50 \mathrm{BCT}(\mathrm{O})$ presents a steady decrease throughout the entire cycling range, with a decrease of $13 \%$ of its initial $d_{33}^{*}$ value after $10^{7}$ cycles.

\section{4 | DISCUSSION}

The unipolar electric cycling caused degradation of electromechanical properties in all three tested compositions. In general, an increase in the bipolar strain hysteresis asymmetry was observed, as well as the development of an internal electric bias field and reduction of the maximum achievable strain in the unipolar strain curves. After thermal annealing, the degradation effects disappeared and the material recovered its initial properties, which indicates that the induced changes were not permanent. Macroscopic pictures of the polished surface of samples were taken before and after cycling to observe possible appearance of cracks. The pictures did not reveal any visible cracks, however, the samples became darker after cycling (Figure S1). This change in color can be an indication for the migration of oxygen vacancies upon unipolar cycling, as observed for acceptor-doped barium titanate. ${ }^{41}$ The color change was also reversed after annealing. Based on these observations, fatigue mechanisms that induce irreversible changes can be excluded. ${ }^{18,20}$ The appearance of an internal bias field and a strong strain asymmetry indicates that the fatigue mechanism for BZT- $x$ BCT is likely related to the internal migration of free charge carriers.

Free charges, moving inside the material under the influence of an external electric field, can accumulate at electrodes or grain boundaries and form local agglomerates. ${ }^{18}$ The surrounding ferroelectric domains reorient toward the charged agglomerates and cause a local polarized region that no longer contributes to the macroscopic strain produced in response to the external electric field. These regions are effectively pinned and result in a positive internal bias field, as well as decreased overall strain output of the material. This mechanism of defect agglomeration has been observed before in $\mathrm{PZT}^{26,27}$ and lead-free ferroelectrics such as BZT- $x \mathrm{BCT}^{33,42}$ and NBT-6BT. ${ }^{43}$ The typical defects expected in oxide perovskite materials are ionic defects from impurities or dopants, oxygen vacancies and defect complexes, resulting from the interaction between other defects. ${ }^{18,44-46}$ Among those, the most mobile defects are the free charge carriers and oxygen vacancies, ${ }^{47}$ which contribute to the fatigue mechanism described previously. ${ }^{13,48,49}$

The electromechanical response of ferroelectrics consists of several contributions, which are typically divided into either intrinsic or extrinsic mechanisms. ${ }^{50}$ Intrinsic processes refer to the inherent lattice distortion of the crystallographic structure, while the extrinsic ones predominantly refer to reversible or irreversible domain wall motions. Both intrinsic and extrinsic contributions can be influenced by proximity to phase transitions and by applied external fields (mechanical and electrical). With a combination of Rayleigh analysis at subcoercive electric fields and macroscopic strain measurements, Gao et al. ${ }^{38}$ investigated the intrinsic and extrinsic piezoelectric contributions in the BZT- $x$ BCT system. The authors concluded that the extrinsic contributions represent the dominant influence to the high piezoelectric response in BZT- $x \mathrm{BCT}$. A quantification of each contribution type to the strain through small-signal measurements as function of electric bias field was presented by Acosta et al. ${ }^{37,51}$ It was revealed that extrinsic processes contribute to $54 \%$ and $64 \%$ of the total room-temperature strain response of the rhombohedral and orthorhombic compositions, respectively. On the other hand, the highest contribution to the strain (73\%) of the tetragonal 60BCT(T) sample was intrinsic in nature, which was attributed to the composition being far from any phase boundaries in the phase diagram. The highest fraction of irreversible contributions was found for the orthorhombic phase.

As described earlier, the defect agglomeration fatigue mechanism has a large influence on the mobility of domains and will thus have a more pronounced effect on samples with a larger fraction of extrinsic contributions. This explains the fact that the most severe degradation was observed for the 50BCT(O) sample (Figures 2-4) since it has the largest extrinsic contributions to its strain response. In contrast, having a large fraction of intrinsic strain contributions made the tetragonal 60BCT(T) sample inherently more resistant to this fatigue mechanism. The above explanation presumes a decrease of the fraction of irreversible contributions to the electromechanical behavior after unipolar cycling, which can be investigated using measurements of the dielectric permittivity at subcoercive AC electric fields and Rayleigh analysis.

\section{1 | Rayleigh analysis}

The Rayleigh law has previously been used to describe the subcoercive piezoelectric and dielectric response of 
ferroelectrics ${ }^{52,53}$ and can be expressed by the following equations:

$$
\begin{gathered}
P(E)=\left(\varepsilon_{\mathrm{r}}^{\prime}(0)+\alpha E_{0}\right) E \pm \frac{1}{2} \alpha\left(E_{0}^{2}-E^{2}\right), \\
\varepsilon_{\mathrm{r}}^{\prime}=\varepsilon_{\mathrm{r}}^{\prime}(0)+\alpha E_{0},
\end{gathered}
$$

where $\varepsilon_{\mathrm{r}}^{\prime}$ is the real part of the relative dielectric permittivity, $\alpha$ is the Rayleigh coefficient, and $E_{0}$ is the electric field amplitude. ${ }^{53,54}$ The Rayleigh region, defined by the range of field amplitudes where the relationship between the $\varepsilon_{\mathrm{r}}^{\prime}$ and $\mathrm{E}$ is linear, is located below the coercive field, where the domain wall density and domain configuration are assumed to be unchanged during the measurement. ${ }^{53}$ The Rayleigh coefficient $\alpha$ correlates the nonlinearity of the polarization response with the extrinsic contributions, assumed here to be predominantly domain wall motions. It can be used as a measure of the irreversibility of domain wall movement. On the other hand, the term $\varepsilon_{\mathrm{r}}^{\prime}(0)$ refers to the permittivity at zero electric field and contains the intrinsic ionic response and the reversible domain wall vibration. ${ }^{53-56}$

Figure 5 depicts the permittivity as a function of subcoercive AC electric field amplitude for all studied compositions both before and after cycling. The slopes of the curves in all cases become lower toward the tetragonal side of the system, which is consistent with previous observations by Gao et al. ${ }^{38}$ and is related to the increased coercive field. The Rayleigh coefficient $\alpha$, taken from the linear regions, and the $\varepsilon_{\mathrm{r}}^{\prime}(0)$ are presented in Table 2. For the $50 \mathrm{BCT}(\mathrm{O})$ and the $60 \mathrm{BCT}(\mathrm{T})$ compositions, $\alpha$ is reduced after cycling, indicating a decrease in the irreversible contributions. This is consistent with the expected defect agglomeration fatigue mechanism. This is most dominant in the orthorhombic sample due to the largest initial extrinsic contributions ${ }^{37}$ and matches well to the fact that its initially large strain was the most heavily reduced by unipolar cycling. From these results, it is inferred that an increased number of defect agglomerates form and act to pin domain walls for this composition. Correspondingly, the strain reduction of the tetragonal composition 60BCT (T) was comparably smaller because its strain response predominantly comes from its intrinsic contribution, that is, lattice distortion. ${ }^{37}$ It is also noted that its decrease in $\alpha$ was of a smaller magnitude with a $\Delta \alpha$ of -315 which represents an $8 \%$ decrease, compared to the $\Delta \alpha$ of -4779 for $50 \mathrm{BCT}(\mathrm{O})$, which represents a decrease of $26 \%$.

Interestingly, $\alpha$ increased after cycling for the $40 \mathrm{BCT}$ (R) composition, indicating enhanced irreversible extrinsic contributions. However, the observed effects of cycling on the strain asymmetry and $\mathrm{E}_{\mathrm{ib}}$ formation are similar in nature to those for the 50BCT(O) and 60BCT(T) compositions. A possible explanation is that the domain configuration changed at some point during the cycling, generating a lower density of domains walls and larger domains, thus increasing the overall fraction of irreversible processes.

\subsection{Domain imaging with piezoresponse force microscopy}

To give further insights into this phenomenon, the domain structures of the three composition before and after cycling were measured with PFM. Figure 6 provides the resulting images for all three compositions, where the lateral and vertical amplitudes are presented on the same spot of the

FIGURE 5 Permittivity as a function of AC electric field amplitude at subcoercive fields for different BZT- $x$ BCT compositions. The measurement frequency was $100 \mathrm{~Hz}$ [Color figure can be viewed at wileyonlinelibrary.com]

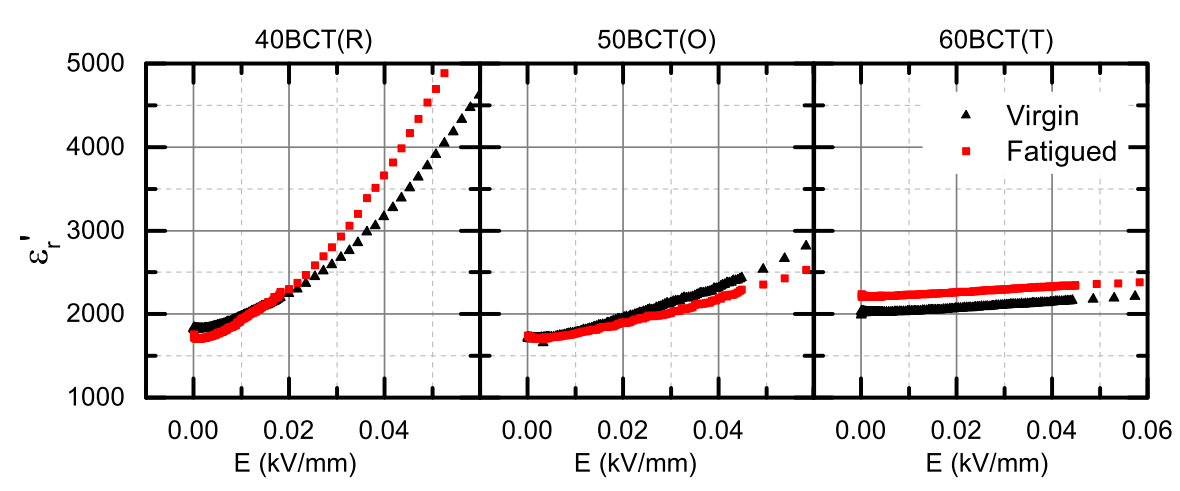

TA B LE 2 Rayleigh coefficient $\alpha$ and $\varepsilon_{\mathrm{r}}^{\prime}(0)$ for investigated compositions before and after cycling

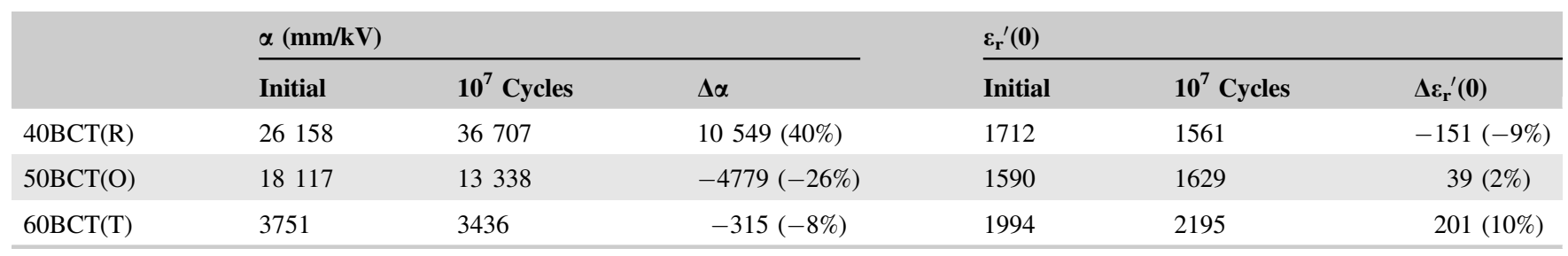




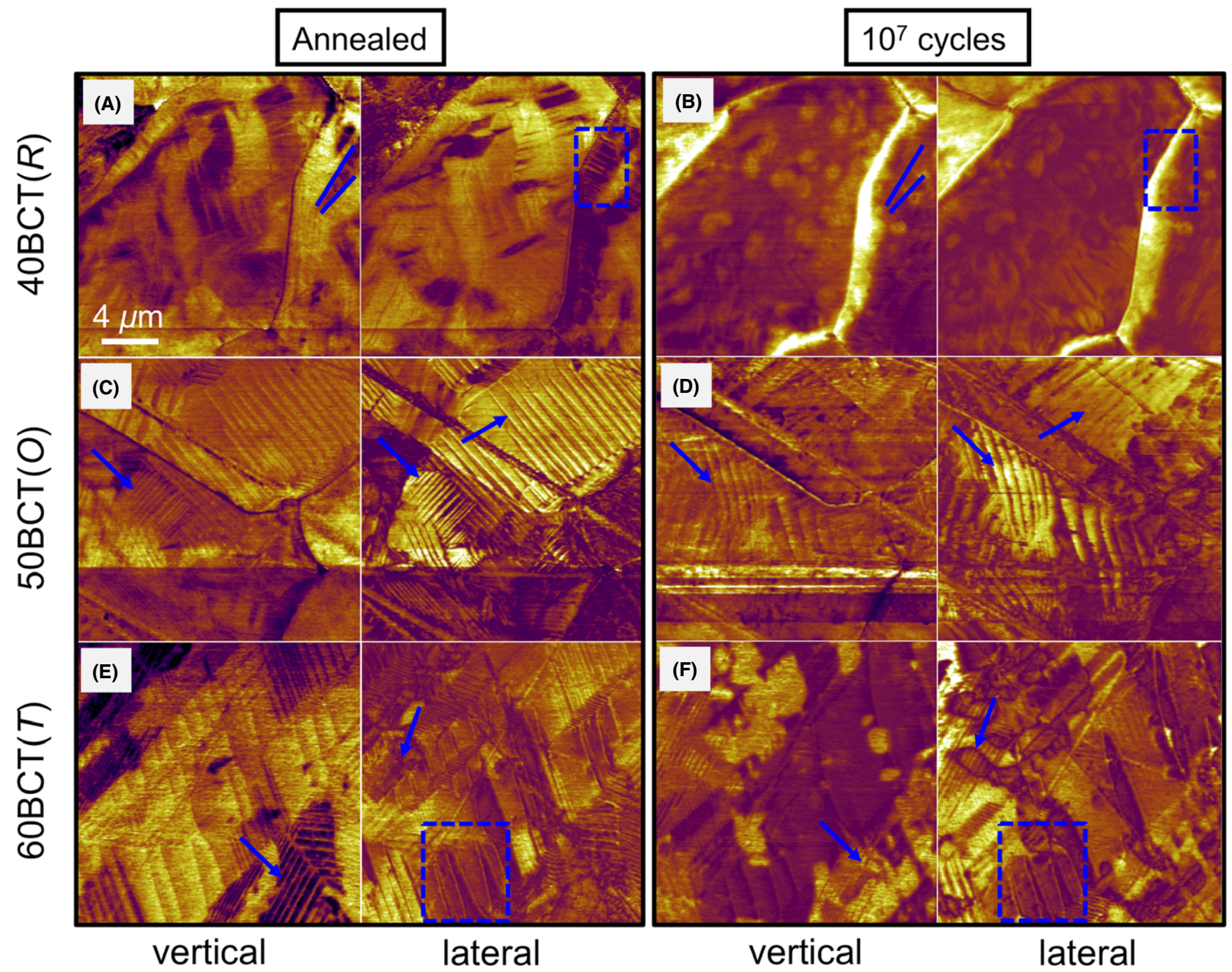

F I G URE 6 Vertical and lateral PFM amplitude images measured before cycling in the annealed state and after $10^{7}$ unipolar cycles: (A) and (B) 40BCT(R), (C) and (D) 50BCT(O), and (E) and (F) 60BCT(T). The areas marked with blue arrows and squares highlight some of the most prominent changes in the domain structures in each image and provide a comparison point between the annealed and fatigued states. Color bars: arbitrary [Color figure can be viewed at wileyonlinelibrary.com]

sample before (in the annealed state) and after cycling. The domain morphologies for all samples were also verified in another area of each sample. The corresponding topography images are shown in the supplementary information (Figure S2).

The domain patterns in $40 \mathrm{BCT}(\mathrm{R})$ are detailed in Figure $6 \mathrm{~A}$ and $\mathrm{B}$. In the annealed state, wedge-shaped domains consisting of lamellar subdomains are observed. The domain morphology changed after cycling and the grains now seem to exhibit a single domain state. The phenomenon of single domain states in BZT- $x$ BCT has been previously observed in TEM studies by Guo et al. ${ }^{57}$ and Zakhozheva et al. ${ }^{58,59}$ The observed single domain developed from a multidomain state upon the application of an in situ external electric field and could be achieved for several compositions with different strengths of nominal poling fields, including 40BCT(R), 50BCT(O), and 60BCT (T). The field amplitudes required to obtain the single domain state were higher for compositions with larger coercive fields, and the domain state was not stable, it reverted to a multidomain state with an increase or removal of the applied field. Our findings indicate that if the single domain state is achieved during unipolar cycling, the initial domain configuration will not be recovered simply by removing the field.

The previously described defect agglomeration fatigue mechanism suggests that domains are pinned by the accumulated defects, decreasing the fraction of irreversible contributions and reducing the electromechanical properties. However, in the case of the 40BCT(R) sample, the domain configuration underwent a dramatic change during cycling from a multidomain to a single domain state. The reduced 
domain wall density is confirmed by the reduction of the zero-field permittivity $\varepsilon_{\mathrm{r}}{ }^{\prime}(0)$ (Table 2). Although the exact reason for this behavior remains unclear, it seems possible that a single domain state, or a state with lower domain wall density, is stabilized by the charged defects agglomerating at the grain boundaries. A detailed in situ microscopic study is needed for understanding of this phenomenon.

In the case of 50BCT(O) (Figure 6C and D) an abundance of lamellar and wedge-shaped domains is observed both in the annealed and in the cycled state. It was previously reported that the initial domain configuration of this composition also contains miniaturized nanodomains within the lamellar arrangement, ${ }^{60,61}$ which we did not observed on our samples. However, the average size of such nanodomains was reported to be $20-100 \mathrm{~nm}$ and thus close to the resolution limit of the PFM analysis. Moreover, these nanodomains were reported to disappear once the material was poled, leaving a micrometer range multidomain configuration. ${ }^{59,62}$ Since the electric field applied for the cycling is sufficiently strong to pole the material during the first cycle, it is assume that the initial nanodomains are not present during the electric cycling and have no influence on the fatigue behavior of this composition.

After unipolar cycling (Figure 6D) the overall configuration of the domains changed and additionally some small or fragmented domains are observed. Although a single domain state has been previously reported also for this composition, ${ }^{57}$ this was not observed in this work. The newly formed, small, fragmented domains are confirmed by the increased parameter $\varepsilon_{\mathrm{r}}{ }^{\prime}(0)$, which is an indicator of increased domain wall density. Domain fragmentation upon cycling was previously observed in PZT using in situ $\mathrm{TEM}^{63}$ and related to decreased irreversible contributions. A decrease of the Rayleigh parameter $\alpha$ upon cycling in our study confirms this effect in the 50BCT(O) sample.

It should also be considered that the composition $50 \mathrm{BCT}(\mathrm{O})$ at room temperature is very close to the phase transition to the tetragonal structure. The applied electric field or even small changes in the temperature could cause a premature phase transition, giving rise to the differences observed in the domain configuration after cycling.

In the case of 60BCT(T) (Figure 6E and F), the lamellar domain configuration found before cycling was still present after cycling. The PFM picture of the cycled state reveals that large domains were unaffected; however, new small fragmented domains were introduced. This is confirmed by the increased parameter $\varepsilon_{\mathrm{r}}{ }^{\prime}(0)$. This behavior is qualitatively the same as observed in the 50BCT $(\mathrm{O})$ sample. The general stability of the overall domain morphology, however, is an indication that intrinsic contributions play a more significant role in this composition.

\section{5 | CONCLUSIONS}

The unipolar fatigue behavior of three BZT- $x$ BCT compositions with different crystallographic structures, that is, 40BCT(R), 50BCT $(\mathrm{O})$, and $60 \mathrm{BCT}(\mathrm{T})$, was evaluated. Domain wall pinning by agglomerated charge carriers is suggested to be the dominant fatigue mechanism in all compositions, and could be removed by a mild thermal annealing $\left(400^{\circ} \mathrm{C}\right.$ for 2 hours). However, the three compositions had distinct responses to the unipolar cycling. The tetragonal composition 60BCT(T) exhibited the best fatigue resistance and after $10^{7}$ unipolar cycles presented a drop of only a $6 \%$ in $\mathrm{P}_{\mathrm{r}}$ and $2 \%$ in $d_{33}^{*}$. The orthorhombic 50BCT (O) was the most affected composition, with a $12 \%$ drop in $\mathrm{P}_{\mathrm{r}}$ and a loss of $13 \%$ for $d_{33}^{*}$. The 40BCT(R) also revealed signs of fatigue, but the final decrease in $\mathrm{P}_{\mathrm{r}}$ and $d_{33}^{*}$ was only $9 \%$ and $3 \%$, respectively.

Results from Rayleigh analysis and PFM were used to relate the fatigue behavior to the different strain mechanisms of each of the three studied compositions. The agglomerated defects decrease the irreversible extrinsic contributions to macroscopic strain, while the intrinsic contributions remain largely unaffected. The high amount of extrinsic contributions to strain made the orthorhombic $50 \mathrm{BCT}(\mathrm{O})$ and rhombohedral 40BCT(R) compositions most susceptible to fatigue during unipolar cycling, evidenced also by the largest reduction of electromechanical properties. The fatigue response of the latter was additionally affected by the formation of a single domain state, which increased the fraction of irreversible contributions and partially compensated the reduction of the electromechanical properties. On the other hand, the high amount of intrinsic contributions to strain made the tetragonal composition $60 \mathrm{BCT}(\mathrm{T})$ more resistant to electric fatigue, resulting in relatively stable electromechanical properties.

\section{ACKNOWLEDGMENTS}

V.R. thanks Patrick Breckner, M.Sc. for experimental support and Maurice Wörner for sample preparation.

\section{REFERENCES}

1. EU-Directive 2011/65/EC: Restriction of the use of Certain Hazardous Substances in Electrical and Electronic Equipment (RoHS). Off J Eur Union. 2011;L174:88-110.

2. Rödel J, Jo W, Seifert KTP, et al. Perspective on the Development of Lead-free Piezoceramics. $J$ Am Ceram Soc. 2009;92:1153-1177.

3. Hong C-H, Kim H-P, Choi B-Y, et al. Lead-free piezoceramics Where to move on? J Materiomics. 2016;2:1-24.

4. Saito Y, Takao H, Tani T, et al. Lead-free piezoceramics. Nature. 2004;432:84-87. 
5. Li J-F, Wang K, Zhu F-Y, et al. (K, Na) $\mathrm{NbO}_{3}$-Based Lead-Free Piezoceramics: fundamental Aspects, Processing Technologies, and Remaining Challenges. J Am Ceram Soc. 2013;96:3677-3696.

6. Malič B, Koruza J, Hreščak J, et al. Sintering of Lead-Free Piezoelectric Sodium Potassium Niobate Ceramics. Materials. 2015;8:8117-8146.

7. Takenaka T, Maruyama K, Sakata K. $\left(\mathrm{Bi}_{1 / 2} \mathrm{Na}_{1 / 2}\right) \mathrm{TiO}_{3}-\mathrm{BaTiO}$ ${ }_{3}$ System for Lead-Free Piezoelectric Ceramics. Jpn J Appl Phys. 1991;30:2236-2239.

8. Jo W, Dittmer R, Acosta M, et al. Giant electric-field-induced strains in lead-free ceramics for actuator applications-status and perspective. J Electroceramics. 2012;29:71-93.

9. Liu W, Ren X. Large Piezoelectric Effect in Pb-Free Ceramics. Phys Rev Lett. 2009;103:257602.

10. Wang D, Jiang Z, Yang B, et al. Phase Diagram and Enhanced Piezoelectric Response of Lead-Free $\mathrm{BaTiO}_{3}-\mathrm{CaTiO}_{3}-\mathrm{BaHfO}_{3}$ System. J Am Ceram Soc. 2014;97:3244-3251.

11. Glaum J, Hoffman M. Electric Fatigue of Lead-Free Piezoelectric Materials. J Am Ceram Soc. 2014;97:665-680.

12. Patterson EA, Cann DP. Piezoelectric properties and unipolar fatigue behavior of KNN-based $\mathrm{Pb}$-free piezoceramics. IEEE Trans Ultrason Ferroelectr Freq Control. 2011;58:1835-1841.

13. Patterson EA, Cann DP. Bipolar piezoelectric fatigue of $\mathrm{Bi}$ $\left(\mathrm{Zn}_{0.5} \mathrm{Ti}_{0.5}\right) \mathrm{O}_{3}-\left(\mathrm{Bi}_{0.5} \mathrm{~K}_{0.5}\right) \mathrm{TiO}_{3}-\left(\mathrm{Bi}_{0.5} \mathrm{Na}_{0.5}\right) \mathrm{TiO}_{3} \mathrm{~Pb}$-free ceramics. Appl Phys Lett. 2012;101:042905.

14. Moulson AJ, Herbert JM. Electroceramics: Materials, Properties, Applications. West Sussex, England: John Wiley \& Sons; 2003.

15. Uchino K. Ferroelectric Devices. CRC Press; 2000.

16. Tagantsev AK, Stolichnov I, Colla EL, et al. Polarization fatigue in ferroelectric films: basic experimental findings, phenomenological scenarios, and microscopic features. $J$ Appl Phys. 2001;90:1387-1402.

17. Lupascu D, Rödel J. Fatigue In Bulk Lead Zirconate Titanate Actuator Materials. Adv Eng Mater. 2005;7:882-898.

18. Lupascu DC. Fatigue in Ferroelectric Ceramics and Related Issues. Heidelberg, Germany: Springer Science \& Business Media; 2013.

19. Wang D, Fotinich Y, Carman GP. Influence of temperature on the electromechanical and fatigue behavior of piezoelectric ceramics. J Appl Phys. 1998;83:5342-5350.

20. Nuffer J, Lupascu DC, Glazounov A, et al. Microstructural modifications of ferroelectric lead zirconate titanate ceramics due to bipolar electric fatigue. J Eur Ceram Soc. 2002;22:2133-2142.

21. Jiang QY, Cross LE. Effects of porosity on electric fatigue behaviour in PLZT and PZT ferroelectric ceramics. J Mater Sci. 1993;28:4536-4543.

22. Zeng FW, Wang H, Lin H-T. Fatigue and failure responses of lead zirconate titanate multilayer actuator under unipolar highfield electric cycling. J Appl Phys. 2013;114:024101.

23. Brennan C. Model of ferroelectric fatigue due to defect/domain interactions. Ferroelectrics. 1993;150:199-208.

24. Nuffer J, Lupascu DC, Rödel J. Damage evolution in ferroelectric PZT induced by bipolar electric cycling. Acta Mater. 2000;48:3783-3794.

25. Glaum J, Granzow T, Schmitt LA, et al. Temperature and driving field dependence of fatigue processes in PZT bulk ceramics. Acta Mater. 2011;59:6083-6092.

26. Balke N, Lupascu DC, Granzow T, et al. Fatigue of Lead Zirconate Titanate Ceramics. I: unipolar and DC Loading. J Am Ceram Soc. 2007;90:1081-1087.
27. Verdier C, Lupascu DC, Rödel J. Unipolar fatigue of ferroelectric lead-zirconate-titanate. J Eur Ceram Soc. 2003;23:1409-1415.

28. Wang H, Cooper TA, Lin H-T, et al. Fatigue responses of lead zirconate titanate stacks under semibipolar electric cycling with mechanical preload. J Appl Phys. 2010;108:084107.

29. Wang H, Wereszczak AA, Lin H-T. Fatigue response of a PZT multilayer actuator under high-field electric cycling with mechanical preload. J Appl Phys. 2009;105:014112.

30. Brandt DRJ, Acosta M, Koruza J, et al. Mechanical constitutive behavior and exceptional blocking force of lead-free BZT $-x$ BCT piezoceramics. J Appl Phys. 2014;115:204107.

31. Coondoo I, Panwar N, Amorín H, et al. Synthesis and characterization of lead-free $0.5 \mathrm{Ba}\left(\mathrm{Zr}_{0.2} \mathrm{Ti}_{0.8}\right) \mathrm{O}_{3}-0.5\left(\mathrm{Ba}_{0.7} \mathrm{Ca}_{0.3}\right) \mathrm{TiO}_{3}$ ceramic. J Appl Phys. 2013;113:214107.

32. Koruza J, Franzbach DJ, Schader F, et al. Enhancing the operational range of piezoelectric actuators by uniaxial compressive preloading. J Phys Appl Phys. 2015;48:215302.

33. Zhang Y, Glaum J, Ehmke MC, et al. High Bipolar Fatigue Resistance of BCTZ Lead-Free Piezoelectric Ceramics. J Am Ceram Soc. 2016;99:174-182.

34. Zhang Y, Glaum J, Ehmke MC, et al. Unipolar Fatigue Behavior of BCTZ Lead-Free Piezoelectric Ceramics. J Am Ceram Soc. 2016;99:1287-1293.

35. Acosta M, Novak N, Jo W, et al. Relationship between electromechanical properties and phase diagram in the $\mathrm{Ba}\left(\mathrm{Zr}_{0.2} \mathrm{Ti}_{0.8}\right)$ $\mathrm{O}_{3}-\mathrm{x}\left(\mathrm{Ba}_{0.7} \mathrm{Ca}_{0.3}\right) \mathrm{TiO}_{3}$ lead-free piezoceramic. Acta Mater. 2014;80:48-55.

36. Bao H, Zhou C, Xue D, et al. A modified lead-free piezoelectric BZT $-x$ BCT system with higher TC. J Phys Appl Phys. 2010; 43:465401.

37. Acosta M, Novak N, Rossetti GAJr, et al. Mechanisms of electromechanical response in $(1-\mathrm{x}) \mathrm{Ba}\left(\mathrm{Zr}_{0.2} \mathrm{Ti}_{0.8}\right) \mathrm{O}_{3}-\mathrm{x}\left(\mathrm{Ba}_{0.7} \mathrm{Ca}_{0.3}\right)$ $\mathrm{TiO}_{3}$ ceramics. Appl Phys Lett. 2015;107:142906.

38. Gao J, Hu X, Zhang L, et al. Major contributor to the large piezoelectric response in $(1-\mathrm{x}) \mathrm{Ba}\left(\mathrm{Zr}_{0.2} \mathrm{Ti}_{0.8}\right) \mathrm{O}_{3}-\mathrm{x}\left(\mathrm{Ba}_{0.7} \mathrm{Ca}_{0.3}\right)$ $\mathrm{TiO}_{3}$ ceramics: domain wall motion. Appl Phys Lett. 2014;104:252909.

39. Kalinin SV, Rodriguez BJ, Jesse S, et al. Vector Piezoresponse Force Microscopy. Microsc Microanal. 2006;12:206-220.

40. Keeble DS, Benabdallah F, Thomas PA, et al. Revised structural phase diagram of $\left(\mathrm{Ba}_{0.7} \mathrm{Ca}_{0.3} \mathrm{TiO}_{3}\right)-\left(\mathrm{BaZr}_{0.2} \mathrm{Ti}_{0.8} \mathrm{O}_{3}\right)$. Appl Phys Lett. 2013;102:092903.

41. Jaffe B, Cook WR, Jaffe HL. Piezoelectric ceramics. Academic Press; 1971.

42. Ehmke MC, Glaum J, Hoffman M, et al. The Effect of Electric Poling on the Performance of Lead-Free $(1-\mathrm{x}) \mathrm{Ba}\left(\mathrm{Zr}_{0.2} \mathrm{Ti}_{0.8}\right) \mathrm{O}_{3}-\mathrm{x}$ $\left(\mathrm{Ba}_{0.7} \mathrm{Ca}_{0.3}\right) \mathrm{TiO}_{3}$ Piezoceramics. J Am Ceram Soc. 2013;96:38053811.

43. Simons H, Glaum J, Daniels JE, et al. Domain fragmentation during cyclic fatigue in $94 \%\left(\mathrm{Bi}_{1 / 2} \mathrm{Na}_{1 / 2}\right) \mathrm{TiO}_{3}-6 \% \mathrm{BaTiO}_{3}$. J Appl Phys. 2012;112:044101.

44. Pöykkö S, Chadi DJ. Dipolar Defect Model for Fatigue in Ferroelectric Perovskites. Phys Rev Lett. 1999;83:1231-1234.

45. Scott JF, Dawber M. Oxygen-vacancy ordering as a fatigue mechanism in perovskite ferroelectrics. Appl Phys Lett. 2000;76:3801-3803.

46. Zhang Y, Glaum J, Ehmke MC, et al. The ageing and de-ageing behaviour of $\left(\mathrm{Ba}_{0.85} \mathrm{Ca}_{0.15}\right)\left(\mathrm{Ti}_{0.9} \mathrm{Zr}_{0.1}\right) \mathrm{O}_{3}$ lead-free piezoelectric ceramics. J Appl Phys. 2015;118:124108. 
47. Raymond MV, Smyth DM. Defects and charge transport in perovskite ferroelectrics. J Phys Chem Solids. 1996;57:1507-1511.

48. Nowotny J, Rekas M. Defect chemistry of $\mathrm{BaTiO}_{3}$. Solid State Ion. 1991;49:135-154.

49. Warren WL, Robertson J, Dimos DB, et al. Transient hole traps in PZT. Ferroelectrics. 1994;153:303-308.

50. Damjanovic D. Contributions to the Piezoelectric Effect in Ferroelectric Single Crystals and Ceramics. J Am Ceram Soc. 2005;88:2663-2676.

51. Acosta M. Strain Mechanisms in Lead-Free Ferroelectrics for Actuators.; Ph.D. Thesis, Technische Universität Darmstadt, Darmstadt, 2015.

52. Damjanovic D, Demartin M. The Rayleigh law in piezoelectric ceramics. J Phys Appl Phys. 1996;29:2057-2060.

53. Hall DA, Stevenson PJ. High field dielectric behaviour of ferroelectric ceramics. Ferroelectrics. 1999;228:139-158.

54. Haugen AB, Morozov MI, Jones JL, et al. Rayleigh analysis of dielectric properties in textured $\mathrm{K}_{0.5} \mathrm{Na}_{0.5} \mathrm{NbO}_{3}$ ceramics. $J$ Appl Phys. 2014;116:214101.

55. Gharb NB, Trolier-McKinstry S. Dielectric nonlinearity of $\mathrm{Pb}$ (Yb1/2Nb1/2)O3-PbTiO3 thin films with 100 and 111 crystallographic orientation. J Appl Phys. 2005;97:064106.

56. Huan Y, Wang X, Li L, et al. Strong domain configuration dependence of the nonlinear dielectric response in $(\mathrm{K}, \mathrm{Na}) \mathrm{NbO} 3-$ based ceramics. Appl Phys Lett. 2015;107:202903.

57. Guo H, Zhou C, Ren X, et al. Unique single-domain state in a polycrystalline ferroelectric ceramic. Phys Rev B. 2014;89:100104.

58. Zakhozheva M, Schmitt LA, Acosta M, et al. In situ electric field induced domain evolution in $\mathrm{Ba}\left(\mathrm{Zr}_{0.2} \mathrm{Ti}_{0.8}\right) \mathrm{O}_{3}-0.3\left(\mathrm{Ba}_{0.7} \mathrm{Ca}_{0.3}\right) \mathrm{TiO}_{3}$ ferroelectrics. Appl Phys Lett. 2014;105:112904.

59. Zakhozheva M, Schmitt LA, Acosta M, et al. Wide Compositional Range In Situ Electric Field Investigations on Lead-Free
$\mathrm{Ba}\left(\mathrm{Zr}_{0.2} \mathrm{Ti}_{0.8}\right) \mathrm{O}_{3}-\mathrm{x}\left(\mathrm{Ba}_{0.7} \mathrm{Ca}_{0.3}\right) \mathrm{TiO}_{3}$ Piezoceramic. Phys Rev Appl. 2015;3:064018.

60. Gao J, Xue D, Wang Y, et al. Microstructure basis for strong piezoelectricity in $\mathrm{Pb}$-free $\mathrm{Ba}\left(\mathrm{Zr}_{0.2} \mathrm{Ti}_{0.8}\right) \mathrm{O}_{3}-\left(\mathrm{Ba}_{0.7} \mathrm{Ca}_{0.3}\right) \mathrm{TiO}_{3}$ ceramics. Appl Phys Lett. 2011;99:092901.

61. Gao J, Zhang L, Xue D, et al. Symmetry determination on Pb-free piezoceramic $0.5 \mathrm{Ba}\left(\mathrm{Zr}_{0.2} \mathrm{Ti}_{0.8}\right) \mathrm{O}_{3}-0.5\left(\mathrm{Ba}_{0.7} \mathrm{Ca}_{0.3}\right) \mathrm{TiO}_{3}$ using convergent beam electron diffraction method. J Appl Phys. 2014;115:054108.

62. Guo H, Voas BK, Zhang S, et al. Polarization alignment, phase transition, and piezoelectricity development in polycrystalline $0.5 \mathrm{Ba}\left(\mathrm{Zr}_{0.2} \mathrm{Ti}_{0.8}\right) \mathrm{O}_{3}-0.5\left(\mathrm{Ba}_{0.7} \mathrm{Ca}_{0.3}\right) \mathrm{TiO}_{3}$. Phys Rev B. 2014;90:014103.

63. Guo H, Liu X, Rödel J, et al. Nanofragmentation of Ferroelectric Domains During Polarization Fatigue. Adv Funct Mater. 2015;25:270-277.

\section{SUPPORTING INFORMATION}

Additional Supporting Information may be found online in the supporting information tab for this article.

How to cite this article: Rojas V, Koruza J, Patterson EA, et al. Influence of composition on the unipolar electric fatigue of $\mathrm{Ba}\left(\mathrm{Zr}_{0.2} \mathrm{Ti}_{0.8}\right) \mathrm{O}_{3^{-}}$ $\left(\mathrm{Ba}_{0.7} \mathrm{Ca}_{0.3}\right) \mathrm{TiO}_{3}$ lead-free piezoceramics. $J \mathrm{Am}$ Ceram Soc. 2017;100:4699-4709. https://doi.org/ $\underline{10.1111 / \text { jace. } 15013}$ 